\title{
Use of polysaccharide hydrogels in drug delivery and tissue engineering
}

\begin{abstract}
Present review article aims to explain use of injectable hydrogels and microspheres derived from natural polysaccharides as drug delivery systems and cell scaffolds. Polysaccharides isolated from natural sources are proved much better than synthetic polymers for making single and composite hydrogels. This paper emphasizes recent developments occurred in use of amphiphilic polysaccharides for biomedical applications. It also explains use of various injectable polysaccharide structure-based hydrogels such as carboxymethyl chitin, hyaluronic acid, PTX-loaded modified hydrogels, Thermosensitive chitosan/dextran-polylactide/glycerophosphate hydrogel, Gellan Gum ,Chitin-CaSO4-nano-fibrin based injectable gel system, nanocomposite hydrogels, chondroitin Sulfate, photo-crosslinked gelatin methacrylate (GelMA) and thermosensitive chitosan/dextran-polylactide/glycerophosphate hydrogels and its functions in engineering cartilage tissue and injectable chemistries. More specifically, this paper high light use of combining analogous matrices with cells/stem cells and biomolecules and multi component approaches for making mimetic constructs. There is a need to develop more advanced designs of polysaccharide-based injectable hydrogels to widen the horizons of clinical drug delivery applications in biomedical engineering and several fields related to pharmaceutics.
\end{abstract}

Keywords: polysaccharides, injectable hydrogels, drug delivery systems, cell scaffolds
Volume 2 Issue 2 - 2017

Ravi Kant Upadhyay
Department of Zoology, DDU Gorakhpur University, India

Correspondence: Ravi Kant Upadhyay, Department of Zoology, DDU Gorakhpur University, Gorakhpur, UP, India, 273009, Email rkupadhya@yahoo.com

Received: February 19, 2017| Published: March 16, 2017
Abbreviations: $\mathrm{CMCH}$, carboxymethyl chitin, HA, hyaluronic acid; PTX, loaded modified hydrogels; TCDPGH, thermosensitive chitosan/dextran-polylactide/glycerophosphate hydrogel, GG, gellan gum; NC, nanocomposite hydrogels; CS, chondroitin sulfate; GelMA, gelatin methacrylate (GelMA) hydrogel

\section{Introduction}

Hydrogels are crosslinked three-dimensional, hydrophilic, polymeric networks, which form both chemical and physical crosslinks when allowed to swell in an aqueous medium. These are capable of imbibing large amounts of water or biological fluids. Hydrogels have several advantages when used as adhesion barriers. These show flexibility, low tissue adhesiveness, biodegradability and are non-toxic to tissues and cells. Furthermore, when compared with preformed hydrogels, injectable hydrogels show superiority as they can fill and cover spaces of any shape and do not require a surgical procedure for implantation than any other biomaterial. ${ }^{1}$ On the basis of structural chemical component used a large variety of hydrogels have been made by cross-linking methods. These have wider applications in pharmaceutical and biomedical fields. Even some of them are able to respond to environmental changes such as temperature, $\mathrm{pH}$, and solvent composition, by changing their dimensions. Hydrogel based on polysaccharides, hydrocolloids, and synthetic polymers are also used for drug delivery. But among the numerous macromolecules that can be used for hydrogel formation, polysaccharides are extremely advantageous compared to synthetic polymers. These are easily procured from plants and more often being produced by recombinant DNA techniques. Polysaccharides are usually non-toxic, biocompatible and show a number of peculiar physico-chemical properties that make them suitable for different applications in drug delivery systems. ${ }^{2}$ Injectable thermosensitive hydrogels are used in various drug delivery systems for their minimally invasive administration and localized drug release. These are workable systems which more efficiently used in cell therapy and tissue regeneration.

Injectable hydrogels and microspheres derived from natural polysaccharides are also used as drug delivery systems and cell scaffolds. These are also found suitable for variety of biomedical applications, such as drug delivery and tissue engineering. ${ }^{2}$ These show tunable mechanical properties, cell deliverability, and facile administration at physiological condition. These show long-term functionality use in osteogensis and hyaline cartilage construction. Interestingly, carbohydrate polymers impart tailorability of desired physicochemical properties and versatile injectable chemistry due to presence of modifiable functional groups. These are used for the development of highly potent biomimetic in situ forming scaffolds. ${ }^{3}$ Chitosan based thermosensitive hydrogels are also prepared which show high mechanical strength. cHA-Dex gel has better chondroprotective and anti-inflammatory effects in rat surgery-induced osteoarthritis than cHA alone. ${ }^{4}$ Tissue adhesion barriers have been developed as films, membranes, knits, sprays, and hydrogels. Adhesions cause serious complications such as postoperative pain, intestinal obstruction, and infertility. Enrichment of hydrogels with inorganic particles desirably improves their suitability for bone regeneration. Because enhanced their mechanical properties also enhance mineralizability, and bioactivity as well as adhesion, proliferation, and differentiation of bone-forming cells, while maintaining injectability. ${ }^{5}$ Polysaccharidebased (amphiphilic) injectable hydrogels are extremely advantageous and have a variety of biomedical applications, such as drug delivery and tissue engineering. These offers easy patient compliance, show tunable mechanical properties, cell deliverability, and facile administration at physiological condition with long-term functionality and hyaline cartilage construction. Injectable hydrogels are also used in cardiac tissue engineering. 
Polysaccharide based hydrogels are used for improvement of structure-based functions in engineering cartilage tissues. These are based on injectable chemistries and made by using combining analogous matrices with cells/stem cells and biomolecules. ${ }^{6}$ Injectable and biodegradable alginate-based composite gel scaffolds doubly integrated with hydroxyapatite (HAp) and gelatin microspheres (GMs) were cross-linked via in situ release of calcium cations. ${ }^{6}$ Chitosan (CS) thermosensitive conductive hydrogels are also designed and generated with a highly porous network of interconnected pores. ${ }^{7}$ Similarly, thermosensitive chitosan/dextran-polylactide/glycerophosphate hydrogel as career for controllable release of bone morphogenetic protein-2 (BMP-2) are also generated. ${ }^{8}$ In these hydrogels BMP-2 is loaded into the microspheres with an average size of around $20 \mu \mathrm{m}$. Consequently, resulting microspheres were then embedded into the gel in order to achieve well-controlled BMP-2 release. Enzymatically cross-linked hydrogels can be formed in situ which permit versatile and selective tethering of bioactive molecules. ${ }^{9}$ A novel biodegradable, thermosensitive and injectable carboxymethyl chitin $(\mathrm{CMCH})$ hydrogel was developed for three-dimensional (3D) cell culture. ${ }^{10}$ In addition, for preparing biodegradable hydrogels sericin, a natural photo luminescent protein from silk, was used to synthesize a hydrazone cross-linked sericin/dextran injectable hydrogel. This hydrogel is biodegradable and biocompatible. It achieves efficient drug loading and controlled release of both macromolecular and small molecular drugs. For local delivery of ibuprofenviaa polygalacturonic acid-hyaluronic acid-based hydrogel reduces the possibility of epidural fibrosis. ${ }^{11}$ PTX-loaded modified hydrogels exhibit 4 times greater antitumor activity compared with Taxol(®). These modified hydrogel systems are used for more efficient sustained release of anticancer drugs that enhance efficiency of anti-tumor therapy. ${ }^{12}$ These gel scaffold exhibit beneficial effects on osteoblast activity and found best local treatment of pathologies involving bone loss. Chitosan gel can increase LV myocardial wall thickness, decrease infarct size. ${ }^{13}$ A good hydrogels form uniform structure and show degradability, robustness, and biocompatibility.

Injectable hydrogels have wider applications in minimally invasive surgical procedures as they efficiently to fill work in defective areas. Injectable electro active hydrogels (eGels) are promising in regenerative medicine and drug delivery. The hydrogel loaded with Doxorubicin significantly suppresses tumor growth. ${ }^{14}$ Further, to endow hydrogels with desirable properties and to incorporate functional nanoparticles in their network composite gel matrixes are also developed. A honey comb composite (gel mixture) is prepared by using the mollusca shell mixture and calcium alginate. This scaffold is found useful to carry cells for application in bone, cartilage, and skin and self tissue regenerative therapies and for tissue engineering. ${ }^{15}$ Thermosensitive injectable chitosan/gelatin, beta glycerophosphate hydrogel is used in preodontal applications. It works as a drug carrier and releases drug that eliminates pathogenic bacteria easily in targeted tissue organ. ${ }^{16}$ Thermosensitive chitosan/gelatin/ beta glycerophosphate disodium salt is used cell encapsulation and cell culture..$^{17}$ These are non-toxic thermo-sensitive polymer systems gels were found highly useful as injectable hydrogels for drug delivery and tissue engineering as they are least invasive to healthy cells. ${ }^{10}$ Similarly, fluorescently labeled injectable hyuronon hydrogels were proved non-invasive in vivo imaging of biomaterials. ${ }^{4}$

In addition, nanocomposite hydrogels are also developed by incorporating PEGylated gold nanorods and paclitaxel-loaded chitosan micelles. ${ }^{18}$ This combination of photothermal-chemotherapy was found effective in tumor control. As synergistically, co-delivered PTX-M shows prolonged tumor retention and perform sustained release of anticancer drug that more efficiently kill the residual tumor cells. It evades the photothermal ablation due to the heterogeneous heating in the tumor region. This combination of photothermalchemotherapy more efficiently suppresses the tumor recurrence in comparison to the photothermal therapy alone. ${ }^{18}$ Similarly, injectable and thermosensitive soluble extracellular matrix and methylcellulose hydrogels are also prepared for stem cell delivery in skin wounds. ${ }^{19}$ These in-situ-forming sECM-MC hydrogels are a promising injectable vehicle for stem cell delivery and tissue regeneration. ${ }^{19}$ A near infrared (NIR) light-responsive supra molecular hydrogel consisting of $\alpha$-cyclo dextrin and poly(ethylene glycol)-modified dendrimer-encapsulated platinum nanoparticles was also developed. This hydrogel showed a photothermo-sensitive degradation to release the entrapped therapeutic agents in an on-demand and dosetunable fashion..$^{20} \mathrm{~A}$ novel nanocomposite injectable hydrogel was prepared by introducing attapulgite (ATP) nano particles into the CS/ GP hydrogels. ${ }^{21}$ ulvan, a sulphated polysaccharide extracted from green seaweeds was used as in-situ hydrogel forming systems for biomedical applications. This injectable thermosensitive hydrogel is proved to be a good drug delivery systems because of their minimally invasive administration and localized drug release. ${ }^{22}$

Further, an advanced double-component injectable formulation (microspheres-loaded hydrogel, CMs-CS-HG) was fabricated that showed good hemocompatibility and histocompatibility, and show no genotoxicity and cytotoxicity to experimental mice. ${ }^{22}$ It shows prolonged drug-release in comparison to single-component CS-HG or CMs. Similarly, a novel drug delivery system that may permit controlled release of $\mathrm{N}$-acetylcysteine (NAC) following intratympanic administration was generated by using two different solutions that attained a hydrogel form within seconds after getting into contact with each other. ${ }^{23}$ The delivery system consisted of an in situ forming thermo responsive poloxamer-based platform combined with $\mathrm{pH}$ responsive polyelectrolyte heparin/chitosan nanocomplexes. This thermo responsive hydrogel with heparin/chitosan nanocomplexes enabled the lowest absorption rate of heparin into systemic circulation and provided heparin concentration above the prophylaxis threshold for 5 days. In addition, in situ gelling thermoresponsive matrix combined with chitosan nanocomplexes was made that is a promising delivery system for heparin, requires less frequent administration during long-term treatment. ${ }^{24}$ Conversion of sulphated polysaccharides obtained from waste algal biomass into high value added material for biomedical applications. ${ }^{25}$

Furthermore, chitosan (CS)/ $\beta$-glycerol phosphate (GP) polymerbased thermosensitive hydrogel were prepared. Chitosan is a natural polymer, and is an important injectable biomedical material that is used in a heat induced gelling system. ${ }^{26}$ Similarly, for prolonged drug release IFN- $\alpha$ (Sumiferon $\left.{ }^{\circledR}\right)$-incorporated hyaluronic acid-tyramine (HA-Tyr) a biodegradable hydrogel system was developed. This IFN- $\alpha$-incorporated HA-Tyr hydrogels + sorafenib have been most effectively inhibited tumor growth on human RCC cells xenografted in nude mice. ${ }^{27}$ Thermogelling chitosan-collagen-bioactive glass nanoparticle hybrids as potential injectable systems for tissue engineering. ${ }^{28}$ It has no toxic effect on the human osteosarcoma cell culture (SAOS) and kidney cells line of human embryo (HEK 293 T). These new composites of gels potentially used as thermo responsive biomaterials for bone-tissue bio-applications. ${ }^{28}$ Similarly, a novel bioinjectable algin-aminocaproic acid (Alg-ACA) tri-stimulus responsive 
thixogel system was also designed which demonstrate temperature and ultrasonication responsiveness. ${ }^{29}$ In addition, few macrocycles such as cyclodextrins and cucurbit[n]urils were also used to increase molecule size and hydrophobicity. These systems are dynamic, show chemical diversity, relative ease of synthesis, and show better ability to interact with biological or synthetic molecules. ${ }^{30}$

An injectable chitosan based hydrogel is used for the treatment of Chronic rhinosinusitis. A small interfering RNA (siRNA) targeting VEGF was used to silence the expression of VEGF, loaded in gel for injection into the sinus cavity. It exhibits long term retention and act as the siRNA carrier. ${ }^{31}$ Algisyl-LVR ${ }^{\mathrm{TM}}$ is used for left ventricular augmentation in patients with dilated cardiomyopathy undergoing open-heart surgery. ${ }^{32}$ Using decellularized skeletal muscle, a simple method was developed for drug absorption after IM injection and drugECM interaction..$^{33}$ An injectable chitosan-based hydrogel $(\mathrm{CH})$ was prepared and is used in surgery removal of tumor. An intraperitoneal injection of macerating solutions from $\mathrm{CH}$ decreased tumor growth and prevented tumor diffusion. ${ }^{20}$ For biomedical use soft tissue fillers are also needed, hence, pullulan and human-like collagen (HLC) based hydrogels were used in combination therapy to restore tissue defects. ${ }^{34}$ HLC/pullulan hydrogels may have therapeutic value as efficient soft tissue fillers, with reduced inflammation, improved cell adhesion and delayed hydrogel degradation.

DOX-loaded PLGA nanoparticles were loaded into the CG hydrogels, for controlled release of DOX from CG hydrogels crosslinked with TG shows a significant potential as a carrier for cell deliver. ${ }^{35}$ Viscosupplementation (VS) is a therapy which is used for osteoarthrosis (OA) consisting of repetitive intra-articular injections of hyaluronic acid (HA). But during injectable therapies cell viability and proliferation is maintained in hydrogels. ${ }^{36}$ More specifically, hybrid hydrogel was composed of HA and reacetylated chitosan, a biopolymer considered to be chondroprotective. Similarly, second formulation consisted of a novel HA polymer grafted with antioxidant molecules (HA-4AR) aiming at decreasing OA oxidative stress and increasing HA retention time in the articulation. ${ }^{37}$ Similarly, a biocompatible conductive biomaterial (PPy-chitosan) that enhances biological conduction in vitro and in vivo was used. Injection of PPychitosan better maintained heart function after myocardial infarction than a nonconductive polymer. ${ }^{38}$ This conductive polymer hydrogel supports cell electrical signaling and improves cardiac function. In addition, hydrogels composed of methylcellulose are considered novel candidate materials for soft tissue reconstruction. For this purpose, methylcellulose modified with functional methacrylate groups is polymerized using an ammonium persulfate (APS)-ascorbic acid(AA) redox initiation system to produce injectable hydrogels with tunable properties. ${ }^{39}$ APS-AA redox-polymerized methylcellulose hydrogels possess properties beneficial for use as soft tissue fillers. Similarly, crosslinking of type II collagen-hyaluronic acid(HA) hydrogel with 1-ethyl-3(3-dimethyl aminopropyl) carbodiimide(EDC) increase the gel stability against collagenase digestion and reduces water uptake in comparison with non-crosslinked gels. This review highlights new polysaccharide polymers, combining analogous matrices with cells/ stem cells and biomolecules and multicomponent approaches for making mimetic constructs for biomedical applications mainly useful in tissue engineering.

\section{Types of materials used}

\section{Carboxymethyl chitin (CMCH) hydrogel}

These thermosensitive injectable carboxymethyl chitin $(\mathrm{CMCH})$ hydrogels are highly useful for biomedical applications mainly in post-operative adhesion prevention, the regeneration of cartilage and central nervous system. ${ }^{10}$ This biodegradable thermosensitive injectable $\mathrm{CMCH}$ hydrogel is used for 3D cell culture and biomedical applications. Bioactive natural chitin was obtained through the "green" process avoiding using organic solvent. ${ }^{10}$

\section{Hyaluronic acid (HA)}

Hyaluronic acid (HA) is highly relevant polysaccharide which is enzymatically cross-linked to hydrogels. ${ }^{40}$ It is a glycosaminoglycan comprised of repeating disaccharide units of $\mathrm{N}$-acetyl-D-glucosamine and D-glucuronic acid. HA is synthesized by hyaluronan synthases and reaches sizes in excess of $2 \mathrm{MDa}$. It plays numerous roles in normal tissues but also has been implicated in inflammatory processes, multiple drug resistance, angiogenesis, tumorigenesis, water homeostasis, and altered viscoelasticity of extracellular matrix. ${ }^{40}$ Cross-linked hyaluronic acid hydrogel (cHA gel) and dexamethasone (Dex) are used to treat knee osteoarthritis (OA). It shows chondroprotective and anti-inflammatory effects. ${ }^{4}$ Further, hybrid HA-PEG hydrogels are composed by using transglutaminasemediated covalent linking of HA moieties with synthetic poly (ethylene glycol) (PEG) macromer. These cytocompatible gels can serve to modulate the cellular phenotype of human mammary cancer epithelial cells as well as mouse myoblasts. ${ }^{40}$ Thiolated hyaluronic acid derivative (HA-SH) are prepared and applied as a three-dimensional scaffold to mimic native extracellular matrix (ECM) for the culture of fibroblasts cells (L929) and chondrocytes. ${ }^{41} \mathrm{HA}-\mathrm{SH}$ hydrogels show intelligent degradation behavior, excellent biocompatibility and convenient operational characteristics. An ibuprofen-conjugated crosslinkable polygalacturonic acid and hyaluronic acid hydrogel was developed for epidural fibrosis prevention. It showed low cytotoxicity and decreased prostaglandin E2production of the lipopolysaccharideinduced RAW264.7 cells. ${ }^{11}$ No doubt HA is a novel building block that can be used in the increasingly varied library of synthetic PEG-based artificial ECMs. As a structural as well as a signaling component it can offer significant potential as an injectable matrix for regenerative and its use may widen clinical applications of hydrogels. ${ }^{9}$ Bio-conjugation of extracellular matrix (ECM) proteins and peptides into matrices, are used for site-specific incorporation of biologically active substances.

\section{PTX-loaded modified hydrogels}

Chitosan based thermosensitive hydrogels are also prepared having high mechanical strength, which was modified by glutaraldehyde (GA) and polyvinyl alcohol (PVA). These are used for intratumoral delivery of paclitaxel (PTX). The modified hydrogel system could achieve sol-gel transition at $35.79 \pm 0.4^{\circ} \mathrm{C}$ and exhibit a 7.03 -fold greater mechanical strength compared with simple chitosan hydrogel. PTX-loaded modified hydrogels exhibited a 3.72-fold greater antitumor activity compared with $\operatorname{Taxol}\left({ }^{\circledR}\right) .{ }^{12}$

\section{Thermosensitive chitosan/dextran-polylactide/glyce- rophosphate hydrogel}

Some delivery carriers with injectable characteristics were also built using the thermosensitive chitosan/dextran-polylactide/ glycerophosphate hydrogel and selected alginate microspheres for the controllable release of bone morphogenetic protein-2 (BMP2). Released BMP-2 also maintained its osteogenesis functions in vivo system. ${ }^{8}$ An injectable micellar supramolecular hydrogel was also developed by using $\alpha$-cyclodextrin $(\alpha-\mathrm{CD})$ and monomethoxy poly(ethylene glycol)-b-poly( $\varepsilon$-caplactone) (MPEG5000-PCL5000) 
micelles. This supramolecular hydrogel is used to deliver hydrophobic anticancer drugs as it enhance the biological activity of encapsulated PTX compared to free PTX, as indicated by in vitro cytotoxicity assay. This micellar supramolecular hydrogel might be a promising injectable drug delivery system for anticancer therapy. ${ }^{42}$

\section{Injectable pullulan hydrogel}

Pullulan was modified through reaction with 2,2,6,6-tetramethyl1-piperidinyloxy (TEMPO) and 1-ethyl-3-(3-dimethylaminopropyl)

Table I Various hydrogel types, biomaterials and their therapeutic applications carbodiimide (EDC) to introduce carboxyl and phenyl groups as crosslinking sites. The modified pullulan hydrogel is an invaluable advance in anti-adhesion agents.

\section{Gellan gum (GG) hydrogel}

Self-gelling, injectable composites of Gellan Gum (GG) hydrogel and two different types of sol-gel-derived bioactive glass (bioglass) particles were used to culture rat mesenchymal stem cells (rMSC) and mineralization. ${ }^{5}$

\begin{tabular}{|c|c|c|c|}
\hline Type of hydrogel & Biomaterial used & Therapeutic application & References \\
\hline $\begin{array}{l}\text { Carboxymethyl chitin } \\
(\mathrm{CMCH}) \text { hydrogel }\end{array}$ & $\mathrm{CMCH}-\mathrm{I} 4$ hydrogels in PBS buffer & $\begin{array}{l}\text { Biodegradable thermosensitive, used } \\
\text { in } 3 \mathrm{D} \text { cell culture and biomedical } \\
\text { applications. }\end{array}$ & 10 \\
\hline Hyaluronic acid(HA) & Hyaluronic acid glycosaminoglycan & $\begin{array}{l}\text { Cross-linked hyaluronic } \\
\text { acid hydrogel(cHA gel) and } \\
\text { dexamethasone(Dex) are used } \\
\text { to treat knee osteoarthritis(OA). } \\
\text { Chondroprotective and anti- } \\
\text { inflammatory effects, respectively. }\end{array}$ & 4 \\
\hline HA-SH hydrogels & Hyaluronic acid-sulfa hydryl hydrogels & $\begin{array}{l}\text { Biocompatible potential and show } \\
\text { clinical application capacity for tissue } \\
\text { engineering and regenerative medicine }\end{array}$ & 41 \\
\hline $\begin{array}{l}\text { PTX-loaded } \\
\text { modified hydrogels }\end{array}$ & PTX-achieve & $\begin{array}{l}\text { High mechanical strength, anti- } \\
\text { tumor therapy efficiency of } \\
\text { anticancer drugs through in situ } \\
\text { tumor injectable administration }\end{array}$ & 12 \\
\hline $\begin{array}{l}\text { Thermosensitive chitosan/ } \\
\text { dextran-polylactide/ } \\
\text { glycerophosphate hydrogel }\end{array}$ & $\begin{array}{l}\text { Chitosan/dextran-polylactide/ } \\
\text { glycerophosphate hydrogel }\end{array}$ & $\begin{array}{l}\text { For the controllable release of bone } \\
\text { morphogenetic protein-2(BMP-2). }\end{array}$ & 8 \\
\hline $\begin{array}{l}a \text {-cyclodextrin }(\alpha-C D) \text { and } \\
\text { monomethoxy poly(ethylene } \\
\text { glycol)-b-poly( }(\varepsilon \text {-caplactone })\end{array}$ & $\begin{array}{l}\text { an injectable micellar supramolecular } \\
\text { hydrogel composed of } \alpha \text {-cyclodextrin }(\alpha-C D) \\
\text { and monomethoxy poly(ethylene glycol)-b- } \\
\text { poly( } \varepsilon \text {-caplactone) (MPEG5000-PCL5000) } \\
\text { micelles }\end{array}$ & $\begin{array}{l}\text { Micellar supramolecular hydrogel might } \\
\text { be a promising injectable drug delivery } \\
\text { system for anticancer therapy }\end{array}$ & 42 \\
\hline Pullulan hydrogels & $\begin{array}{l}\text { Modified through reaction } \\
\text { with 2,2,6,6-tetramethyl-I- } \\
\text { piperidinyloxy(TEMPO) and I-ethyl-3- } \\
\text { (3dimethylaminopropyl)carbodiimide (EDC) } \\
\text { to introduce carboxyl and phenyl groups as } \\
\text { crosslinking sites }\end{array}$ & $\begin{array}{l}\text { Hydrogels, injectable hydrogels can fill } \\
\text { and cover spaces of any shape and do } \\
\text { not require a surgical procedure for } \\
\text { implantation }\end{array}$ & $\mathrm{I}$ \\
\hline $\begin{array}{l}\text { Chitin-CaSO4-nano-fibrin } \\
\text { based injectable gel system }\end{array}$ & $\begin{array}{l}\text { Calcium sulfate( } \mathrm{CaSO} 4) \text {, an excellent } \\
\text { biodegradable bone forming agent }\end{array}$ & $\begin{array}{l}\text { Potential of injectable chitin-CaSO4- } \\
\text { nFibrin gel for osteo-regeneration via } \\
\text { enhanced angiogenesis. }\end{array}$ & $\begin{array}{l}\text { 44, Arun } \\
\text { Kumar et } \\
\text { al. }^{43}\end{array}$ \\
\hline Gellan Gum(GG) hydrogel & $\begin{array}{l}\text { Sol-gel-derived bioactive glass (bioglass) } \\
\text { particles were analyzed in the wet state }\end{array}$ & $\begin{array}{l}\text { Self-gelling, injectable composites used } \\
\text { in regenerative therapies. }\end{array}$ & 5 \\
\hline (POEGMA) & $\begin{array}{l}\text { (POEGMA) and rigid rod-like cellulose } \\
\text { nanocrystals(CNCs) }\end{array}$ & $\begin{array}{l}\text { Nanocomposite hydrogels based on } \\
\text { poly(oligoethylene glycol methacrylate)): }\end{array}$ & 44 \\
\hline Chondroitin Sulfate hydrogel: & Chondroitin Sulfate & $\begin{array}{l}\text { Injectable pullulan/chondroitin sulfate } \\
\text { composite hydrogels presented here } \\
\text { are expected to be useful biomaterial } \\
\text { scaffold for regenerating cartilage tissue }\end{array}$ & 45 \\
\hline GelMA hydrogel and CM-SIN & GelMA hydrogel and CM-SIN & $\begin{array}{l}\text { Drug and cell delivery system in } \\
\text { cartilage tissue engineering. }\end{array}$ & 47 \\
\hline $\begin{array}{l}\text { Photo-crosslinked gelatin } \\
\text { methacrylate (GelMA) } \\
\text { hydrogel }\end{array}$ & gelatin methacrylate (GelMA) hydrogel & $\begin{array}{l}\text { Injectable drug and cell delivery system } \\
\text { in cartilage tissue engineering }\end{array}$ & 46 \\
\hline
\end{tabular}




\section{Chitin-CaSO4-nano-fibrin based injectable gel system}

Calcium sulfate (CaSO4), an excellent biodegradable bone forming agent that is an ideal choice as additive in gels. Chitin-CaSO4-nanofibrin based injectable gel system which shows improved rheology and angiogenic potential. ${ }^{43}$

\section{Nano composite hydrogels}

Nano composite hydrogels based on poly (oligoethylene glycol methacrylate) (POEGMA) and rigid rod-like cellulose nanocrystals $(\mathrm{CNCs})$ are also made by in situ-gelling. These nanocomposite hydrogels based on poly (oligoethylene glycol methacrylate) and rigid rod-like cellulose nanocrystals $(\mathrm{CNCs})$ showed dramatic enhancements in mechanical properties. As they show faster gelation rates, decreased swelling ratios, and increased stability versus hydrolysis. These POEGMA-CNC nanocomposite hydrogels have various biomedical applications including tissue engineering scaffolds for stiffer tissues or platforms for cell growth. ${ }^{44}$

\section{Chondroitin sulfate hydrogel}

Further, enzymatically cross-linked injectable hydrogel system comprising carboxymethyl pullulan-tyramine (CMP-TA) and chondroitin sulfate-tyramine (CS-TA) conjugates was developed in presence of both horseradish peroxidase (HRP) and hydrogen peroxide (H2O2) for cartilage tissue engineering (CTTE). The HRP crosslinking method makes this injectable system feasible, minimally invasive and easily translatable for regenerative medicine applications. These injectable pullulan/chondroitin sulfate composite hydrogels were found highly useful biomaterial scaffold for regenerating cartilage tissue. ${ }^{45}$ Furthermore, covalent hydrogels were prepared by using polysaccharides via the Schiff' base reaction. For cross-linking of hydrogels water soluble carboxymethyl chitosan (CMC) and oxidized chondroitin sulfate (OCS) were used. This, composite $\mathrm{CMs} / \mathrm{gel}$ scaffolds exhibited lower swelling ratio and slower degradation rate than the control hydrogel without CMs. It shows high encapsulation of bovine articular chondrocytes in vitro and can be used cell delivery system in cartilage tissue engineering. ${ }^{46}$

\section{Photo-crosslinked gelatin methacrylate (GelMA) hy- drogel}

A combination of CM-SIN and GelMA (GelMA hydrogels and chitosan microspheres) was also developed. Drug sinomenium (SIN) was encapsulated by chitosan microspheres (CM-SIN) and photo-crosslinked gelatin methacrylate (GelMA) hydrogel. This combination of GelMA hydrogel and CM-SIN stopped progression of surgically induced OA. It could be used as a promising strategy for treating patients with osteoarthritis. ${ }^{47}$

\section{Conclusion}

Polysaccharide injectable hydrogel gel systems are feasible, minimally invasive and easily translatable for regenerative medicine applications. These form hydrophilic, polymeric networks, with chemical or physical cross-links, capable of imbibing large amounts of water or biological fluids. Among the numerous macromolecules polysaccharides are extremely advantageous compared to synthetic polymers being widely present in living organisms and often being produced by recombinant DNA techniques. Coming from renewable sources, polysaccharides also have frequently economical advantages over synthetic polymers. Polysaccharides are usually non-toxic, biocompatible and show a number of peculiar physico-chemical properties that make them suitable for different applications in drug delivery systems. No doubt hydrogel network prepared from polysaccharides is highly useful in drug delivery and have so many future manifestations in tissue engineering. There are categories of injectable hydrogel systems such as CMs embedded CMC-OCS hydrogels used in cartilage tissue engineering. Similarly, injection of the combination of GelMA hydrogel and CM-SIN could be a promising strategy for treating patients with osteoarthritis. The HRP crosslinking method makes this injectable system feasible, minimally invasive and easily translatable for regenerative medicine applications. In addition, nanocomposite hydrogels based on poly (oligoethylene glycol methacrylate) (POEGMA) and rigid rod-like cellulose nanocrystals (CNCs). Also have several biomedical use. Further, in situ-gelling nanocomposite hydrogels based on poly(oligoethylene glycol methacrylate) (POEGMA) and rigid rod-like cellulose nanocrystals (CNCs) showed increased gelation rate, swelling kinetics, mechanical properties, and hydrogel stability that can be readily tailored. Bioglass (GG hydrogels) promoted attachment of rat mesenchymal stem cells (rMSC) and mineralization. Supramolecular chemistry has emerged as an important technique for the formation of biomaterials, including nano- and microparticles and hydrogels. Enrichment of hydrogels with inorganic particles improves their suitability for bone regeneration by enhancing their mechanical properties, mineralizability, and bioactivity as well as adhesion, proliferation, and differentiation of bone-forming cells, while maintaining injectability. Low aggregation and homogeneous distribution maximize particle surface area, promoting mineralization, cell-particle interactions, and homogenous tissue regeneration. Micellar supramolecular hydrogel might be a promising injectable drug delivery system for anticancer therapy.

\section{Acknowledgements}

None.

\section{Conflict of interest}

The author declares no conflict of interest.

\section{References}

1. Bang S, Lee E, Ko YG, Injectable pullulan hydrogel for the prevention of postoperative tissue adhesion. Int J Biol Macromol. 2016;87:155-162.

2. Thambi T, Phan VH, Lee DS. Stimuli-sensitive injectable hydrogels based on polysaccharides and their biomedical applications. Macromol Rapid Commun. 2016;37(23):1881-1896.

3. Radhakrishnan J, Subramanian A, Krishnan UM, et al. Injectable and 3D bioprinted polysaccharide hydrogels: from cartilage to osteochondral tissue engineering. Biomacromolecules. 2017;18(1):1-26.

4. Zhang Z, Wei X, Gao J, et al. Intra-Articular Injection of Cross-Linked Hyaluronic Acid-Dexamethasone Hydrogel Attenuates Osteoarthritis: An Experimental Study in a Rat Model of Osteoarthritis. Int J Mol Sci. 2016;17(4):411.

5. Gorodzha S, Douglas TE, Samal SK, et al. High-resolution synchrotron $\mathrm{X}$-ray analysis of bioglass-enriched hydrogels. J Biomed Mater Res A. 2016;104(5):1194-201.

6. Yan J, Miao Y, Tan H, et al. Injectable alginate/hydroxyapatite gel scaffold combined with gelatin microspheres for drug delivery and bone tissue engineering. Mater Sci Eng C Mater Biol Appl. 2016;63:274-284.

7. Baei P, Jalili Firoozinezhad S, Rajabi Zeleti S, et al. Electrically conductive gold nanoparticle-chitosan thermosensitive hydrogels for cardiac tissue engineering. Mater Sci Eng C Mater Biol Appl. 2016;63:131-141. 
8. Zhu Y, Wang J, Wu J, Zhang J, et al. Injectable hydrogels embedded with alginate microspheres for controlled delivery of bone morphogenetic protein-2. Biomed Mater. 2016;11(2):025010.

9. Ranga A, Lutolf MP, Hilborn J, et al. Hyaluronic acid hydrogels formed in situ by transglutaminase-catalyzed reaction. Biomacromolecules. 2016;17(5):1553-1560.

10. Liu H, Liu J, Qi C, et al. Thermosensitive injectable in-situ forming carboxymethyl chitin hydrogel for three-dimensional cell culture. Acta Biomater. 2016;35:228-237.

11. Lin CY, Peng HH, Chen MH, et al. Ibuprofen-conjugated hyaluronate/ polygalacturonic acid hydrogel for the prevention of epidural fibrosis. $J$ Biomater Appl. 2016;30(10):1589-1600.

12. Jiang Y, Meng X, Wu Z, et al. Modified chitosan thermosensitive hydrogel enables sustained and efficient anti-tumor therapy via intratumoral injection. Carbohydr Polym. 2016;144:245-253.

13. Henning RJ, Khan A, Jimenez E. Chitosan hydrogels significantly limit left ventricular infarction and remodeling and preserve myocardial contractility. J Surg Res. 2016;201(2):490-497.

14. Wang B, Jakus AE, Baptista PM, et al. Functional Maturation of Induced Pluripotent Stem Cell Hepatocytes in Extracellular Matrix-A Comparative Analysis of Bioartificial Liver Microenvironments. Stem Cells Transl Med. 2016;5(9):1257-1267.

15. You HJ, Li J, Zhou C, et al. A honeycomb composite of mollusca shell matrix and calcium alginate. Colloids Surf B Biointerfaces. 2016;139:100-106.

16. Pakzad Y, Ganji F. Thermosensitive hydrogel for periodontal application: in vitro drug release, antibacterial activity and toxicity evaluation. J Biomater Appl. 2016;30(7):919-929.

17. Heidari Keshel S, Rostampour M, et al. Derivation of epithelial-like cells from eyelid fat-derived stem cells in thermosensitive hydrogel. $J$ Biomater Sci Polym Ed. 2016;27(4):339-350.

18. Zhang N, Xu X, Zhang X, et al. Nanocomposite hydrogel incorporating gold nanorods and paclitaxel-loaded chitosan micelles for combination photothermal-chemotherapy. Int J Pharm. 2015;497(1-2):210-221.

19. Kim EJ, Choi JS, Kim JS, et al. Injectable and thermosensitive soluble extracellular matrix and methylcellulose hydrogels for Stem cell delivery in skin wounds. Biomacromolecules. 2016;17(1):4-11.

20. Wang H, Song F, Chen Q, et al. Antitumor and antimetastasis effects of macerating solutions from an injectable chitosan-based hydrogel on hepatocarcinoma. J Biomed Mater Res A. 2016;103(12):3879-3885.

21. Wang Q, Chen D. Synthesis and characterization of a chitosan based nanocomposite injectable hydrogel. Carbohydr Polym. 2016;136:12281237.

22. Dang Q, Liu C, Wang Y, et al. Characterization and biocompatibility of injectable microspheres-loaded hydrogel for methotrexate delivery. Carbohydr Polym. 2016;136:516-526.

23. Ciftci Z, Deniz M, Yilmaz I, et al. In vitro analysis of a novel controlled release system designed for intratympanic administration of $\mathrm{N}$-acetylcysteine: a preliminary report. Am J Otolaryngol. 2015;36(6):786-793.

24. Matanović MR, Grabnar PA, Voinovich D, et al. Development and preclinical pharmacokinetics of a novel subcutaneous thermoresponsive system for prolonged delivery of heparin. Int J Pharm. 2015;496(2):583592.

25. Morelli A, Betti M, Puppi D, et al. Design, preparation and characterization of ulvan based thermosensitive hydrogels. Carbohydr Polym. 2016;136:1108-1117.
26. Lu S, Yang Y, Yao J, et al. Exploration of the nature of a unique natural polymer-based thermosensitive hydrogel. Soft Matter. 2016;12(2):492499 .

27. Ueda K, Akiba J, Ogasawara S, et al. Growth inhibitory effect of an injectable hyaluronic acid-tyramine hydrogels incorporating human natural interferon- $\alpha$ and sorafenib on renal cell carcinoma cells. Acta Biomater. 2016;29:103-111.

28. Moreira CD, Carvalho SM, Mansur HS, et al. Thermogelling chitosan-collagen-bioactive glass nanoparticle hybrids as potential injectable systems for tissue engineering. Mater Sci Eng C Mater Biol Appl. 2016;58:1207-1216

29. Chejara DR, Mabrouk M, Badhe RV, et al. A bio-injectable algin-aminocaproic acid thixogel with tri-stimuli responsiveness. Carbohydr Polym. 2016;135:324-333.

30. Rodell CB, Mealy JE, Burdick JA. Supramolecular guest-host interactions for the preparation of biomedical materials. Bioconjug Chem. 2016;26(12):2279-2289.

31. Cao C, Yan C, Hu Z, et al. Potential application of injectable chitosan hydrogel treated with siRNA in chronic rhinosinusitis therapy. Mol Med Rep. 2015;12(5):6688-6694.

32. Lee RJ, Hinson A, Bauernschmitt R, et al. The feasibility and safety of Algisyl-LVR ${ }^{\mathrm{TM}}$ as a method of left ventricular augmentation in patients with dilated cardiomyopathy: initial first in man clinical results. Int $J$ Cardiol. 2015;199:18-24.

33. Wassenaar JW, Boss GR, Christman KL. Decellularized skeletal muscle as an in vitro model for studying drug-extracellular matrix interactions. Biomaterials. 2015;64:108-114.

34. Li X, Xue W, Liu Y, et al. HLC/pullulan and pullulan hydrogels: their microstructure, engineering process and biocompatibility. Mater Sci Eng C Mater Biol Appl. 2016;58:1046-1057.

35. Tormos CJ, Abraham C, Madihally SV. Improving the stability of chitosan-gelatin-based hydrogels for cell delivery using transglutaminase and controlled release of doxycycline. Drug Deliv Transl Res. 2015;5(6):575-584.

36. Priyadarshani $\mathrm{P}, \mathrm{Li} \mathrm{Y}$, Yang $\mathrm{S}$, et al. Injectable hydrogel provides growth-permissive environment for human nucleus pulposus cells. $J$ Biomed Mater Res A. 2015;104(2):419-426.

37. Kaderli S, Viguier E, Watrelot Virieux D, et al. Efficacy study of two novel hyaluronic acid-based formulations for viscosupplementation therapy in an early osteoarthrosic rabbit model. Eur J Pharm Biopharm. 2015;96:388-395

38. Mihic A, Cui Z, Wu J, et al. A conductive polymer hydrogel supports cell electrical signaling and improves cardiac function after implantation into myocardial infarct. Circulation. 2015;132(8):772-784.

39. Gold GT, Varma DM, Taub PJ, et al. Development of crosslinked methylcellulose hydrogels for soft tissue augmentation using an ammonium persulfate-ascorbic acid redox system. Carbohydr Polym. 2015;134:497-507.

40. Hemshekhar M, Thushara RM, Chandranayaka S, et al. Emerging roles of hyaluronic acid bioscaffolds in tissue engineering and regenerative medicine. Int J Biol Macromol. 2016;86:917-928.

41. Bian S, He M, Sui J, et al. The self-crosslinking smart hyaluronic acid hydrogels as injectable three-dimensional scaffolds for cells culture. Colloids Surf B Biointerfaces. 2016;140:392-402.

42. Fu C, Lin X, Wang J, et al. Injectable micellar supramolecular hydrogel for delivery of hydrophobic anticancer drugs. J Mater Sci Mater Med. 2016;27(4):73. 
43. Arun Kumar R, Sivashanmugam A, Deepthi S, et al. Nano-fibrin stabilized $\mathrm{CaSO} 4$ crystals incorporated injectable chitin composite hydrogel for enhanced angiogenesis \&amp; osteogenesis. Carbohydr Polym. 2016;140:144-153.

44. De France KJ, Chan KJ, Cranston ED, et al. Enhanced mechanical properties in cellulose nanocrystal-poly(oligoethylene glycol methacrylate) injectable nanocomposite hydrogels through control of physical and chemical cross-linking. Biomacromolecules. 2016;17(2):649-660.

45. Chen F, Yu S, Liu B, et al. An injectable enzymatically crosslinked carboxymethylated pullulan/chondroitin sulfate hydrogel for cartilage tissue engineering. Sci Rep. 2016;6:20014.
46. Fan M, Ma Y, Tan H, et al. Covalent and injectable chitosan-chondroitin sulfate hydrogels embedded with chitosan microspheres for drug delivery and tissue engineering. Mater Sci Eng C Mater Biol Appl. 2017;71:67-74.

47. Chen $\mathrm{P}, \mathrm{Xia} \mathrm{C}, \mathrm{Mei} \mathrm{S}$, et al. Intra-articular delivery of sinomenium encapsulated by chitosan microspheres and photo-crosslinked GelMA hydrogel ameliorates osteoarthritis by effectively regulating autophagy. Biomaterials. 2016;81:1-13. 